\title{
Artificial Protein Hydrogel Materials
}

\author{
W. A. PETKA ${ }^{1}$, J. L. HARDEN ${ }^{2}$, J. K. SAKATA ${ }^{1,3}$, and D. A. TIRRELL ${ }^{3}$ \\ ${ }^{1}$ Department of Polymer Science and Engineering \\ University of Massachusetts, Amherst, MA 01003 \\ ${ }^{2}$ Department of Chemical Engineering \\ Johns Hopkins University, Baltimore, MD 21218 \\ ${ }^{3}$ Division of Chemistry and Chemical Engineering \\ California Institute of Technology, Pasadena, CA 91125
}

\begin{abstract}
Recombinant DNA methods were used to create a new class of artificial proteins that undergo reversible gelation in response to changes in $\mathrm{pH}$ or temperature. These proteins consist of terminal $\alpha$-helical "leucine zipper" domains flanking a central, water-soluble polyelectrolyte segment. The formation of coiled-coil aggregates of the terminal domains in near-neutral $\mathrm{pH}$ solution triggers formation of a polymer hydrogel, with the central polyelectrolyte segment retaining solvent and preventing precipitation of the chains. Dissociation of the coiled-coil aggregates through elevation of $\mathrm{pH}$ or temperature causes dissolution of the gel and a return to the viscous behavior characteristic of a polymer solution. The $\mathrm{pH}$ and temperature range of the hydrogel state and its viscoelastic properties may be systematically varied through precise changes of the length, composition and charge density of the terminal and central blocks. Such control is of value in designing hydrogels with predetermined physical properties and makes these biosynthetic triblock copolymer systems attractive candidates for use in molecular and cellular encapsulation and in controlled reagent delivery.
\end{abstract}

\section{Introduction}

Protein engineering of new functional macromolecules promises to be a valuable tool in creating novel biopolymers for speciality materials applications [1]. The synthesis of macromolecules via biological routes provides direct, simultaneous control of stereochemistry, sequence, and molecular weight. This in turn provides a method for making materials that have both the functional properties of natural proteins and the structural properties of synthetic polymers. We illustrate this approach here through the design of hydrogel materials based on a new class of artificial proteins that undergo controlled, reversible gelation in response to changes in $\mathrm{pH}$ or temperature.

Hydrogels are a special class of functional gel materials that respond to different types of stimuli in aqueous environments [2]. They can be designed, for instance, to swell or shrink under certain physiological conditions and they can be used to encapsulate cells, drugs, and other molecules for site specific use [3]. As an alternative to chemically prepared hydrogels, our interests are to synthesize sequence-controlled protein-based hydrogels that are $\mathrm{pH}$ and temperature reversible as a result of tunable molecular recognition and association processes. These systems are model triblock associating polymers [4] in which the associating blocks, containing both ionic and hydrophobic groups, are engineered independently from the non-interacting, soluble spacer blocks.

\section{Protein Design}

The triblock architecture of our synthetic telechelic proteins, illustrated in Figure 1A, consists of two helical "leucine zipper" domains separated by a central, water-soluble poly- 
electrolyte domain. The modular nature of the design allows independent modification of the terminal associating blocks and the central solubilizing block. Furthermore, the terminal and central blocks may be separately expressed for the purposes of control studies.
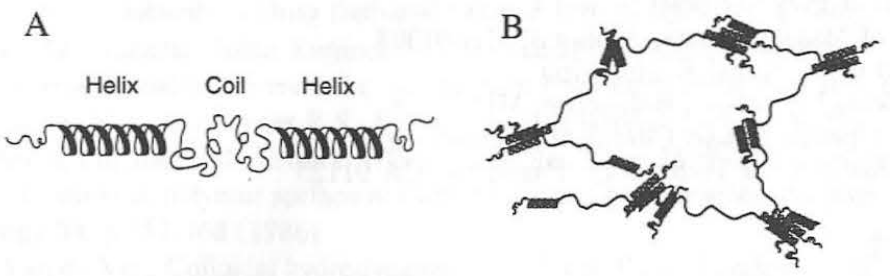

Figure 1: (A) Sketch of the helix-coil-helix protein architecture;

(B) Proposed physical gelation of the associating triblock proteins.

The leucine zipper motif occurs widely in nature and is characterized by a repetitive heptad pattern, ( $a b c d e f g$ ), where $a$ and $d$ are hydrophobic amino acids (typically leucine, particularly at position $d$ ) and the residues at positions $e$ and $g$ are usually charged [5]. Over a range of $\mathrm{pH}$ and temperature, such peptides adopt a $7_{2}$ helical conformation that places the hydrophobic residues at $a$ and $d$ on a single face of the helix, as shown in Figure 2 . Helix-helix aggregation is driven by attractive interactions between such hydrophobic faces. The ionizable residues at $e$ and $g$ are immediately adjacent to the $a$ and $d$ residues in the helical state. The $\mathrm{pH}$-dependent, inter- and intra-molecular interactions between ionizable residues modulate the stability of these aggregates. The gelation of our triblock proteins, sketched in Figure 1B, is governed by these competing interactions, leading to a tunable gel-sol transition.
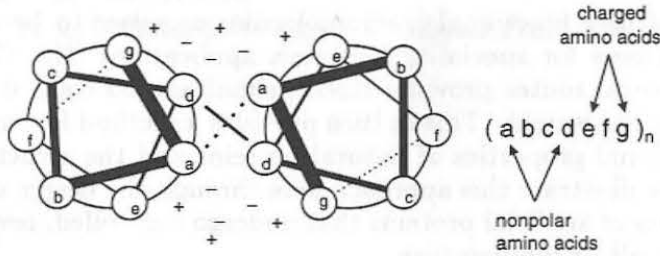

Figure 2: Sketch of a helix-helix binding configuration for leucine zipper sequences.

Systematic studies of peptides of the generic leucine zipper type have shown that aggregation number [6], dimerization specificity [7], and aggregate stability [8] can be widely manipulated through control of amino acid sequence architecture and length. The results of these studies suggest that the network junctions in gels of triblock proteins with leucine zipper end groups should also be subject to such control. In this paper, we will limit our discussion to two types of leucine zipper domains, each comprising six heptad repeats. In the acidic helix (designated Helix $\mathrm{A}_{\mathrm{A}}$, nine of the twelve $e$ and $g$ positions are filled by glutamic acid residues, whereas in the basic helix (designated Helix $_{\mathrm{B}}$ ), nine of the twelve $e$ and $g$ positions are filled by lysine residues. The choice of hydrophobic residues at positions $a$ and $d$ was based on the $a / d$ residue pattern of the Jun oncogene product [9], while the 
residues occupying the $b, c$, and $f$ positions were chosen using a database of the the most probable amino acids at positions $a-g$ in naturally occurring coiled-coil proteins [10].

The polyelectrolyte spacer domain is based on the alanine-glycine-rich sequence $\left[(\mathrm{AG})_{3} \mathrm{PEG}\right]_{n}$, a water soluble motif that lacks a regular secondary structure $[11]$. The sequence, denoted $\mathrm{C}_{n}$, contains one glutamic acid per nine amino acid residues, which is primarily responsible for its solubility in aqueous environments. A variety of different triblock proteins have been synthesized from the $\mathrm{Helix}_{\mathrm{A}}, \mathrm{Helix}_{\mathrm{B}}$, and $\mathrm{C}_{n}$ constructs [12]. We restrict our discussion here to spacer segments with $n=10$ and two triblock motifs. One triblock, designated $\mathrm{AC}_{10} \mathrm{~A}$, has an acidic Helix $\mathrm{A}_{\mathrm{A}}$ at each end, while the second, designated $\mathrm{AC}_{10} \mathrm{~B}$, has an acidic $\mathrm{Helix}_{\mathrm{A}}$ at one end and a basic $\mathrm{Helix}_{\mathrm{B}}$ at the other end. Each triblock motif has a terminal cysteine residue, allowing for the end-linking of two triblocks via a disulfide bond.

\section{Molecular Characterization}

The $\mathrm{AC}_{10} \mathrm{~A}$ and $\mathrm{AC}_{10} \mathrm{~B}$ proteins were synthesized by bacterial expression of the corresponding artificial genes using standard molecular biology techniques $[12,13]$. Helix ${ }_{A}$, Helix $_{\mathrm{B}}$, and $\mathrm{C}_{10}$ spacer coil proteins were also synthesized as controls. The detailed amino acid sequences for these proteins are given in Ref. [12]. The molecular weights and compositions of these artificial proteins were determined by reversed phase high performance liquid chromatography, amino acid compositional analysis, and matrix assisted laser desorption mass spectrometry [12].

The secondary structures of the triblock proteins and their component parts in dilute $(5 \mu \mathrm{M})$ aqueous salt solutions $\left(\mathrm{pH} 7.4,10 \mathrm{mM} \mathrm{NaH} \mathrm{PO}_{4}, 150 \mathrm{mM} \mathrm{NaCl}\right)$ were determined by circular dichroism spectroscopy (CD) over the wavelength range 195-250 $\mathrm{nm}[12,13]$. At room temperature, $\mathrm{CD}$ measurements confirmed the disordered coil character of the $\mathrm{C}_{10}$ spacer block (as indicated by a global minimum in mean residue ellipticity at $201 \mathrm{~nm}$ ), and the helical structure of the leucine zipper blocks in both $\mathrm{AC}_{10} \mathrm{~A}$ and isolated $\mathrm{Helix}_{\mathrm{A}}$ sequences (as indicated by local minima in the mean residue ellipticity at 208 and $222 \mathrm{~nm}$ ). Analysis of the CD results using a variety of protein structure algorithms showed that the helicity of the coiled-coil end domains of the triblock is not reduced by introducing the central disordered coil spacer block. Furthermore, dilute solutions of either $\mathrm{AC}_{10} \mathrm{~A}$ or $\mathrm{Helix}_{\mathrm{A}}$ exhibited thermal unfolding transitions, with the temperature of the transition midpoint $\left(T_{m}\right)$ rising in each case from around $30^{\circ} \mathrm{C}$ at $\mathrm{pH} 11$ to greater than $80^{\circ} \mathrm{C}$ at $\mathrm{pH} 6$, due to the progressive neutralization of the predominantly acidic charged residues with decreasing $\mathrm{pH}$. The abrupt increase in $T_{m}$ between $\mathrm{pH} 7.5$ and 6 is characteristic of coiled-coil peptides with positions $e$ and $g$ occupied predominantly by acidic residues. The thermal unfolding transition of leucine zipper sequences depends strongly on sequence architecture. In contrast to $\mathrm{Helix}_{\mathrm{A}}$, the Helix $\mathrm{B}_{\mathrm{B}}$ unfolding transition occurs at relatively high temperature at high $\mathrm{pH}$ and progressively lower temperature with decreasing $\mathrm{pH}$ [12].

\section{Hydrogel Behavior}

The gel-sol behavior of $\mathrm{AC}_{10} \mathrm{~A}$ in concentrated solution may be anticipated from its dilute solution behavior. In a low $\mathrm{pH}$ solution, the glutamic acid side chains $\left(\mathrm{pK}_{a} \simeq 4.3\right)$ are protonated and uncharged, resulting in stable coiled-coil aggregates. Furthermore, protonation of the glutamic acid residues in the central $\mathrm{C}_{10}$ domain causes its collapse into a hydrophobic globule. Hence, precipitation of $\mathrm{AC}_{10} \mathrm{~A}$ should occur at low $\mathrm{pH}$. With increasing $\mathrm{pH}$, progressive deprotonation of the glutamic acid residues occurs, resulting first in swelling of the collapsed spacer block and eventually, at higher $\mathrm{pH}$, in the dissociation of coiled-coil end domains due to the repulsive electrostatic interactions between predominantly negatively charged endblocks. Thus, in a certain range of $\mathrm{pH}$, the $\mathrm{AC}_{10} \mathrm{~A}$ protein 
should form a thermoreversible physical gel, where the reversibility of the gelled state results from thermal dissociation of the coiled-coil domains. At sufficiently high $\mathrm{pH}$, a viscous solution of predominantly non-associated proteins results. This proposed scenario is borne out by simple visual observation: Aqueous solutions of $\mathrm{AC}_{10} \mathrm{~A}$, which flow readily at $\mathrm{pH}$ 10 , exhibit no visible flow on a time scale of hours upon acidification to $\mathrm{pH} 7$. Subsequent acidification below $\mathrm{pH} 6$ results in precipitation of the protein from solution.

The gel-sol behavior of $\mathrm{AC}_{10} \mathrm{~B}$ is quite different from that of $\mathrm{AC}_{10} \mathrm{~A}$, due to the different inter-molecular electrostatic interactions between acidic and basic leucine zipper domains. Whereas electrostatic interactions between $\mathrm{Helix}_{\mathrm{A}}$ domains become progressively repulsive with increasing $\mathrm{pH}$, interactions between $\mathrm{Helix}_{\mathrm{A}}$ and $\mathrm{Helix}_{\mathrm{B}}$ are attractive at moderate and high $\mathrm{pH}$. Hence, one expects the gelled state to occupy a wider range of $\mathrm{pH}$ for $\mathrm{AC}_{10} \mathrm{~B}$ than for $\mathrm{AC}_{10} \mathrm{~A}$. We note that aqueous solutions of Helix $\mathrm{A}_{\mathrm{A}}, \mathrm{Helix}_{\mathrm{B}}$, and $\mathrm{C}_{10}$ do not gel at any $\mathrm{pH}$, indicating that both aggregating terminal groups and a spacer block are necessary constituents of a gel-forming protein of this type.

We have used diffusing wave spectroscopy (DWS) [14], a non-invasive optical technique based on dynamic light scattering in the multiple scattering limit, to investigate the $\mathrm{pH}$ and temperature dependence of protein gelation. With DWS, the viscoelastic behavior of a complex fluid is determined by monitoring the thermally induced fluctuations of a dilute suspension of added scattering particles [14]. The time averaged mean-square displacement (MSD) of the tracer particles as a function of time, $\left\langle\Delta r^{2}(t)\right\rangle$, may be obtained from the dynamic intensity autocorrelation function of light that is multiply scattered from these tracer particles [15]. The MSD of the tracer particles contains essential information about the viscoelastic properties of the embedding medium. In a purely viscous liquid the tracer particles may freely diffuse, resulting in a MSD that increases linearly with time; while for an elastic medium, the amplitude of the tracer particle fluctuations is limited, resulting in a plateau in the MSD. In a viscoelastic medium, the MSD will show some aspects of each limiting behavior. More precisely, it can be shown that the MSD of the tracer particles is related to the shear creep compliance $J(t)$ of an incompressible embedding medium by $J(t)=\left(\pi a / k_{B} T\right)<\Delta r^{2}(t)>$, where $a$ is the radius of a tracer particle and $k_{B} T$ is the thermal energy. Equivalently, the complex shear modulus $G^{*}(\omega)$ may be obtained from $\left.G^{*}(\omega)=k_{B} T /\left(i \omega \pi a<\Delta \tilde{r}^{2}(\omega)\right\rangle\right)$, where $\left\langle\Delta \tilde{r}^{2}(\omega)\right\rangle$ is the Fourier transform of $<\Delta r^{2}(t)>$.

The gel-sol transition in solutions of $\mathrm{AC}_{10} \mathrm{~A}$ or $\mathrm{AC}_{10} \mathrm{~B}$ can be identified by the emergence of a plateau in the MSD of a previously liquid-like sample in response to changes in $\mathrm{pH}$ or temperature. The plateau modulus $G_{p}$ in the gel state is then related to the plateau $<\Delta r^{2}(t)>_{p}$ in the MSD by $G_{p}=k_{B} T /\left(\pi a<\Delta r^{2}(t)>_{p}\right)$. The MSD measured for $\mathrm{AC}_{10} \mathrm{~A}$ protein solutions $\left[5 \%(\mathrm{w} / \mathrm{v})\right.$ in $10 \mathrm{mM}$ Tris $\left.\mathrm{HCl}, 23^{\circ} \mathrm{C}\right]$ at $\mathrm{pH} 8.0,8.8$, and 9.5 are shown in Figure 3A. At pH 8.0, the MSD exhibits a plateau characteristic of an elastic gel with a plateau modulus $G_{p} \simeq 200 \mathrm{~Pa}$ near $\omega=100 \mathrm{~s}^{-1}$. Such gel behavior is observed for protein concentrations above about $4 \%(\mathrm{w} / \mathrm{v})$. At $\mathrm{pH} 8.8$, this plateau region is reduced to an inflection point, whereas at $\mathrm{pH} 9.5$, the MSD increases with time as in a viscous liquid. These data show a progressive transformation from elastic to viscoelastic to viscous behavior and suggest that the gel point for $\mathrm{AC}_{10} \mathrm{~A}$ lies between $\mathrm{pH} 8.0$ and 9.5.

The thermal dependence of gelation for $\mathrm{AC}_{10} \mathrm{~A}$ was investigated by cycling a $5 \%$ gel through a series of temperatures ranging from $23^{\circ} \mathrm{C}$ to $55^{\circ} \mathrm{C}$ at $\mathrm{pH} 7.8$. The MSD versus time at various temperatures is plotted in Figure 3B. The solid curves in Figure $3 \mathrm{~B}$ show the data obtained during the heating portion of the cycle; the dashed curve shows the MSD after cooling from $55^{\circ} \mathrm{C}$ back to $23^{\circ} \mathrm{C}$. These data show that as the temperature was increased to $55^{\circ} \mathrm{C}$, the gel progressively became a fluid. Furthermore, the gel state was recovered upon cooling back to $23^{\circ} \mathrm{C}$ from the fluid state at $55^{\circ} \mathrm{C}$, although the recovery is not complete after one cycle [13]. 

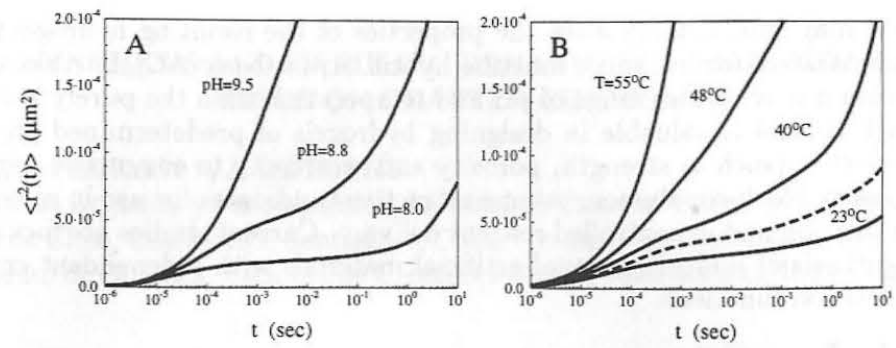

Figure 3: Log-linear plots of $\left\langle\Delta r^{2}(t)\right\rangle$ vs time for $\mathrm{AC}_{10} \mathrm{~A}$ solutions [5\% (w/v) in $10 \mathrm{mM}$ Tris $\mathrm{HCl}$ : (A) $\mathrm{pH} 8.0,8.8$, and 9.5 at $23^{\circ} \mathrm{C}$; and (B) at $\mathrm{pH} 7.8$, collected upon heating from $23^{\circ} \mathrm{C}$ (bottom solid curve) to $55^{\circ} \mathrm{C}$ (top solid curve) and upon cooling to $23^{\circ} \mathrm{C}$ (dashed curve).

The dependence of gel behavior on the architecture of the leucine zipper terminal groups was studied for $5 \%$ solutions of $\mathrm{AC}_{10} \mathrm{~A}$ and $\mathrm{AC}_{10} \mathrm{~B}$ in $10 \mathrm{mM}$ Tris $\mathrm{HCl}$ for a range of $\mathrm{pH}$ and temperature. Comparative $\mathrm{MSDs}$ for $\mathrm{AC}_{10} \mathrm{~A}$ and $\mathrm{AC}_{10} \mathrm{~B}$ are shown in Figure 4. Figure $4 \mathrm{~A}$ shows MSDs for samples at moderate $\mathrm{pH}$ and relatively high temperature ( $\mathrm{pH} 7.8$ and $55^{\circ} \mathrm{C}$ ). Note that while the $\mathrm{AC}_{10} \mathrm{~A}$ sample has fully melted at this temperature, the corresponding $\mathrm{AC}_{10} \mathrm{~B}$ sample is still quite solid. Figure $4 \mathrm{~B}$ shows $\mathrm{MSD}$ s for samples at room temperature and relatively high $\mathrm{pH}\left(\mathrm{pH} 9.5\right.$ and $\left.23^{\circ} \mathrm{C}\right)$. In this case, the $\mathrm{AC}_{10} \mathrm{~B}$ sample is a relatively strong gel $\left(G_{p} \simeq 500 \mathrm{~Pa}\right)$, even though the corresponding $\mathrm{AC}_{10} \mathrm{~A}$ sample has dissociated into a viscous solution. Thus, there is a dramatic dependence of the gel-sol transition and the corresponding gel properties on the architecture of the associating block.
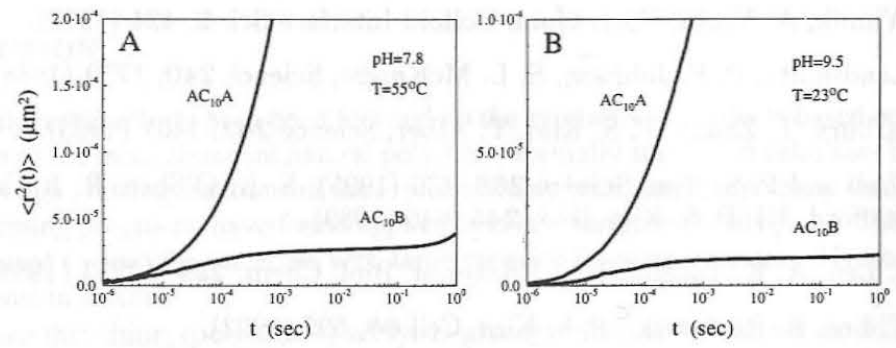

Figure 4: Log-linear plot of $<\Delta r^{2}(t)>$ vs time for $\mathrm{AC}_{10} \mathrm{~A}$ and $\mathrm{AC}_{10} \mathrm{~B}$ solutions $[5 \%(\mathrm{w} / \mathrm{v})$ in $10 \mathrm{mM}$ Tris $\mathrm{HCl}$ ]: (A) at $\mathrm{pH} 7.8$ and $55^{\circ} \mathrm{C}$ and $(\mathrm{B})$ at $\mathrm{pH} 9.5$ and $23^{\circ} \mathrm{C}$.

\section{Conclusions}

The gelation behavior of our artificial proteins illustrates the advantages of biological synthesis and assembly processes used in combination with concepts drawn from macromolecular materials science. The triblock protein design introduced here preserves the dimerization function of the leucine zipper domain, but turns that function toward entirely new objectives. Protein-protein recognition, which in nature might lead to DNA binding, now results in the formation of switchable hydrogels. The biosynthetic approach allows precise and independent control of the length, composition, and charge density of the associating endblocks and the soluble spacer domain. Through variation of such molecular 
properties, one may substantially alter the properties of the resulting hydrogel materials. We have demonstrated, for instance, that the hybrid acidic-basic $\mathrm{AC}_{10} \mathrm{~B}$ triblocks exist in the gel state over a much wider range of $\mathrm{pH}$ and temperature than the purely acidic $\mathrm{AC}_{10} \mathrm{~A}$ triblocks. Such control is valuable in designing hydrogels of predetermined physical and biological properties (such as strength, porosity and sensitivity to enzymatic degradation) and makes these triblock copolymer systems attractive candidates for use in molecular and cellular encapsulation and in controlled reagent delivery. Current studies are focused on the design and synthesis of multi-functional artificial materials with independent encapsulant binding and gelation functions.

\section{Acknowledgments}

We acknowledge support by grants from the National Science Foundation and the U.S. Army Natick Research Development and Engineering Center (to D.A. Tirrell). We thank K. P. McGrath for useful discussions and assistance in the early phase of this project, and D. Wirtz and J. H. van Zanten for the use of DWS facilities.

\section{References}

[1] Protein-Based Materials, edited by K. McGrath and D. Kaplan (Birkhauser, Boston, MA 1997).

[2] J.-M. Guenet, Thermoreversible Gelation of Polymers and Biopolymers (Academic Press, London, 1992).

[3] Hydrogels and Biodegradable Polymers for Bioapplications, edited by R. M. Ottenbrite, S. J. Huang, and K. Park (American Chemical Society, Washington, D.C., 1996).

[4] M. A. Winnik, A. Yekta, Curr. Opin. Colloid Interface Sci. 2, 424 (1997).

[5] W. H. Landshultz, P. F. Johnson, S. L. McKnight, Science 240, 1759 (1988).

[6] P. B. Harbury, T. Zhang, P. S. Kim, T. Alber, Science 262, 1401 (1993).

[7] K. J. Lumb and P. S. Kim, Science 268, 436 (1995); E. K. O'Shea, R. Rutkowski, W. F. Stafford. III, P. S. Kim, ibid. 245, 646 (1989).

[8] S. Y. M. Lau, A. K. Taneja, R. S. Hodges, J. Biol. Chem. 259, 13253 (1984).

[9] E. K. O’Shea, R. Rutkowski, P. S. Kim, Cell 68, 699 (1992).

[10] A. Lupas, M. V. Dyke, J. Stock, Science 252, 1162 (1991).

[11] K. P. McGrath, M. J. Fournier, T. L. Mason, D. A. Tirrell, J. Am. Chem. Soc. 114, 727 (1992).

[12] W. A. Petka, PhD thesis, University of Massachusetts, Amherst, 1997.

[13] W. A. Petka, J. L. Harden, K: P. McGrath, D. Wirtz, and D. A. Tirrell, Science 281, 389 (1998).

[14] D. A. Weitz, D. J. Pine, in Dynamic Light Scattering; The Method and Some Applications, edited by W. Brown (Clarendon, Oxford, 1993), p. 719.

[15] T. G. Mason, D. A. Weitz, Phys. Rev. Lett. 74, 1252 (1995);

T. G. Mason, H. Gang, D. A. Weitz, J. Mol. Struct. 81, 383 (1996). 